\title{
Experimental and theoretical studies of pumps of irrigation pumping stations
}

\author{
Oleg Glovatskiy ${ }^{1,2}$, Rustam Ergashev ${ }^{2}$, Jaloliddin Rashidov ${ }^{2}$, Naira Nasyrova, ${ }^{2}$ and Boybek \\ Kholbutaev $^{3}$ \\ ${ }^{1}$ Research Institute of Irrigation and Water Problems, Tashkent, 100187, Uzbekistan \\ ${ }^{2}$ Tashkent Institute of Irrigation and Agricultural Mechanization Engineers, Tashkent, 100000, \\ Uzbekistan \\ ${ }^{3}$ Jizzakh Polytechnical Institute, Jizzakh, 130100,Uzbekistan
}

\begin{abstract}
The article presents the results of a study of the wear of individual pump elements, a decrease in their efficiency and significant losses. The nonidentical operation of the extreme and middle pumps was established. The uneven approach of water to the extreme units created reverse flows of water in the suction pipes, work with vibrations of modes close to cavitation. The modernization of the pumps improved the cavitation characteristics due to the elimination of the cavitation center at the impeller inlet in the area of the driven disk, which increased the reliability and durability of the pump. Diagnostics of irrigation pumps made it possible to substantiate proposals for the modernization of their main units and operating modes. The distribution of pumps according to the general level of vibration is shown, indicating the direction at which the maximum value of vibration displacement was recorded and the results of a comparative measurement of the vibration of the units of the pumping station-1 of the Karshi Main Channel (KMC).Studies of the pumps of large irrigation pumping stations have shown a reduction in vibration and an improvement in basic operating parameters. With the participation of the authors, new complex methods of operation are being developed, which allow increasing its reliability by predicting the technical condition.
\end{abstract}

\section{Introduction}

In the XXI century, the systems of pumping water in the Republic of Uzbekistan amount to more than 2.4 million hectares (50\% of irrigated arable land). The area of non-irrigated farmland: non-irrigated arable land, pastures, hayfields is more than 2.3 million hectares. Pumps are widely used in these areas. The Republic of Uzbekistan is the most pumped republic, more than half of the pumping capacity of the entire Central Asian region is concentrated here. The experience of operating pumping stations (PS) is of great importance for developing countries, where climatic and hydrological conditions are similar $[1,2]$.

At the present stage, reliable and efficient operation has become one of the most 
important requirements for many types of pumps. Therefore, the issues of reducing the intensity of noise and vibration of pumps at the source of their origin have acquired particular urgency $[3,4]$.

A review of works devoted to improving the operating modes of centrifugal pumps showed that the developed mathematical models describe individual processes and most of them are not suitable for developing the most profitable operating modes; the problem of unsteady water movement in the PS has not been fully resolved $[5,6]$.

\section{Methods}

The studies of the authors have established the action of axial forces on the working bodies of the pumps. If the coefficients are established empirically for a certain pump operating in a given mode, then the derived equations can be used to determine the maximum permissible suction height or the minimum required head for cavitation conditions. The results of our experiments confirm the correctness of this reasoning.

\section{Results and discussion}

To develop effective ways to increase efficiency, reduce noise and vibration, it has become necessary to study the influence on them of energy parameters, design and technological factors and operating conditions of vane pumps. However, many of these factors defied calculation. The task was complicated by the specificity of measuring the vibroacoustic characteristics, for the determination of which it was necessary to create special conditions for testing the pumps excluding the influence of noise and vibration disturbances $[7,8]$.

The limitation of noise and vibration of pumps is dictated by many circumstances, including direct technical requirements, operating conditions, the need to reduce the harmful effects of vibration and noise. Noise and vibration levels are general indicators of pump reliability and quality. Modern pump research is a complex task of significant technical complexity, requiring a large number of experiments [9,10].

The flow path of the pump serves to convert part of the kinetic energy of the flow leaving the wheel into pressure. In this case, inevitable energy losses occur, the minimum of these losses usually determines the position of the maximum efficiency of the pump as a whole.

The phenomena of shock and flow separation, pulsations of pressure and velocities at the exit from the wheel and in flow elements, navigation processes are the main reasons for the occurrence of oscillatory processes in the pump, which are recorded in the form of vibrations and airborne noise. Modern calculation methods pursue the goal of obtaining the maximum energy performance of the pump at the design operating mode [11, 12]. However, this design approach does not always provide minimal pump losses.

The above information substantiates the need for research to improve the operating modes of PS with blade units.

The authors carried out research on the PS "Babotag" with horizontal double-entry pumps DVL-1400x900 with a flow rate of $4.44 \mathrm{~m}^{3} / \mathrm{s}$; head $78.28 \mathrm{~m}$; Efficiency $86.0 \%$; cavitation headroom (NPSHR) $10.2 \mathrm{~m}$.

The modernization of the pump improved its cavitation characteristics by eliminating the center of cavitation at the inlet to the impeller in the area of the driven disk, which increased the reliability and durability of the pump $[3,13]$.

In order to improve the cavitation characteristics, at the suggestion of the authors, the fairing on the shaft side can be made with a cavity in which the guide baffles are located and fixed in the body by means of ribs. 
The axial force $\mathrm{F}_{1 \mathrm{a}}$ acts on the wheel as the difference in external pressure on the side discs of the wheel on the right and left sides.

$$
\begin{gathered}
F_{1 a}=2 \pi \int_{r_{s p}}^{r_{2}}\left(p-p_{i n}\right) r d r \\
F_{1 a}=2 \pi \int_{r_{s p}}^{r_{2}}\left\{p_{2}-\gamma \frac{u_{2}^{2}}{8 g}\left[1-\frac{r^{2}}{r_{2}^{2}}\right]\right\} d r
\end{gathered}
$$

Internal pressure on the sidewalls will be balanced. From the inlet side, the pressure on the side wall will be less, since the pressure in the inlet $p_{s p}$ from the inside is always less than the pressure from the outside. The pressure from the outside is determined by the rotation $u_{2}$ of the fluid at a speed in the gap between the wheel wall and the housing at a radius $r_{2}$. Its value is determined by the formula:

$$
p=p_{2}-\frac{\mu_{2}^{2}}{8 g}\left(1-\frac{r^{2}}{r_{2}^{2}}\right)
$$

The pressure in the gap, counted from the outer diameter, drops more slowly than in the wheel, since the fluid in the gap rotates at half the angular velocity. The pressure forces on the lateral surfaces at radii larger than the entry radius $r_{\mathrm{sp}}$ are mutually balanced. Unbalanced pressure forces occur over the area defined by the inlet diameter.

For the established deviations of hydrodynamic quantities from their steady-state values, a mathematical model of the unsteady flow of an incompressible medium has been developed in one of the following forms $[6,9]$.

$$
\begin{aligned}
& Q=\int_{0}^{t} \omega_{Q p}\left(t^{\prime}\right) p_{1-2}\left(t-t^{\prime}\right) d t^{\prime} \\
& p_{1-2}=\int_{0}^{t} \omega_{p Q}\left(t^{\prime}\right) Q\left(t-t^{\prime}\right) d t^{\prime},
\end{aligned}
$$

where $Q$ - pump flow;

$p_{1-2}$ - pressure difference at the inlet and outlet of the flow path;

$\omega_{Q p}(t)$ - impulse transient function describing the change in flow rate $Q$ over time with a change in pressure $p_{1-2}$ difference;

$\omega_{p Q}(t)$ - a similar function describing the change in pressure difference over time with a change in flow $Q$.

For known functions $\omega_{Q p}(t)$ either $\omega_{p Q}(t)$ the dependences $Q$ or $p_{1-2}$ on time are found from the integral relations (4), (5) changes in time, respectively $p_{1-2}$ or $Q$. It is 
more difficult to experimentally determine impulse transient functions than transient functions caused by a step change in the specified values; therefore, instead of relations (4) or (5), we use the related relations:

$$
Q=\frac{d}{d t} \int_{0}^{t} h_{Q p}\left(t^{\prime}\right) p_{1-2}\left(t-t^{\prime}\right) d t^{\prime}
$$

or

$$
p_{1-2}=\frac{d}{d t} \int_{0}^{t} h_{p Q}\left(t^{\prime}\right) Q\left(t-t^{\prime}\right) d t^{\prime} .
$$

in which $h_{Q p}(t)$ and $h_{p Q}(t)$ are the transient functions of the flow rate $Q$ or the pressure difference $p_{1-2}$.

Axial force arises from the change in the amount of movement of water in the axial direction. This force, as reactive, can be calculated from the difference in the momentum in the axial direction at the outlet and inlet to the impeller $\bar{c}_{1 a}$.

$$
F_{2 a}=-\frac{\gamma Q^{\prime}}{g}\left(\bar{c}_{2 a}-\bar{c}_{1 a}\right)
$$

The axial speed at the exit from the wheel $\bar{c}_{2 a}=0$, therefore the axial force $\mathrm{P}_{2 \mathrm{a}}$ is found by the formula

$$
P_{2 a}=\frac{\gamma Q^{\prime}}{g} c_{1 a}
$$

This force is the reaction force of the inflowing jet. Forces $F_{1 a}$ and $F_{2 a}$ are directed in different directions. Total axial force $F_{a}$; will be found as the difference of these forces

$$
F_{a}=F_{1 a}-F_{2 a}
$$

The axial thrust loads the bearings, complicating and heavier the pump design. This effort must be reduced, i.e. relieve the wheel from axial force. The wheel is sealed at the front and rear. On the rear side, at a radius smaller than the radius of the seal, there are holes called unloading holes $[14,15]$. The pressures on the front and back sides are equalized. This method of axial thrust unloading is used on many pumps during retrofitting at the impeller inlet (Fig. 1). 


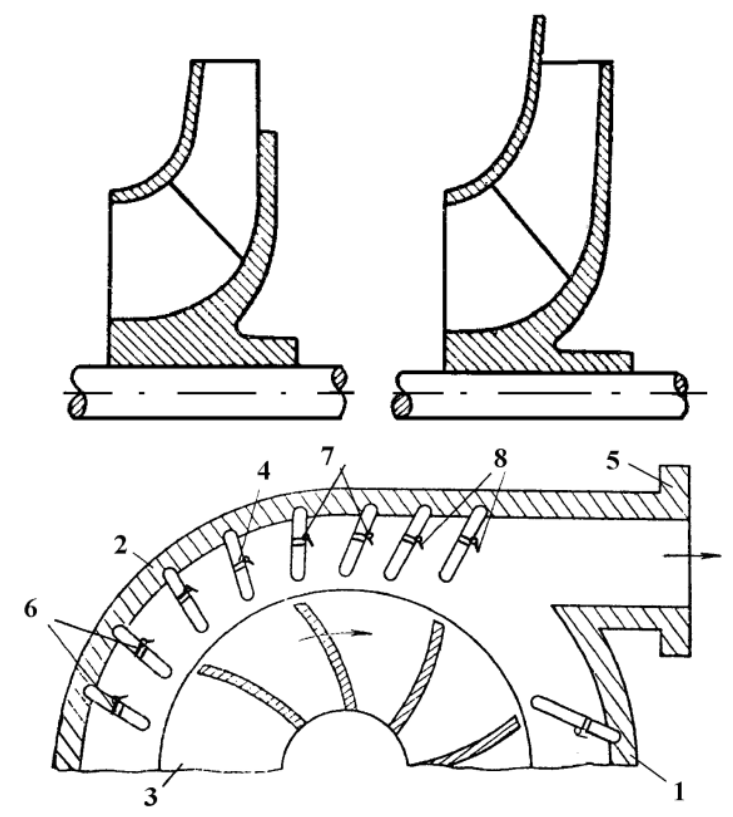

Fig. 1. Balancing the axial force with a new impeller design

When the impeller 3 rotates, the liquid enters the spiral chamber 2, through the baffles 4 , as a result of which normally closed valves 8 open on the hinges 7 , which bypass the liquid with significant energy into the discharge pipe 5 . When vortex zones of unsteady operation processes are formed, especially when the pump is stopped, reverse flows close valves 8 , eliminating eddies and flow separation from the inner surface of the housing 1.

The constructive execution of holes 6 with a diameter of $5-10 \%$ of the inner diameter of the discharge pipe 5 at a distance of $40-50 \%$ from the place of attachment of the baffles 4 in the body 1 provides, on the basis of model studies, the passage of $90-95 \%$ of the volumes of reverse flows, which have features of the wall at a distance $40-50 \%$ of the inner surface of the body 1 .

The baffles 4 create effective swirling of the flow over the entire open section of the volute 2 and the conversion of the additional velocity head of the vortex motion of the liquid into pressure.

At high pump flow rates, the growth of the hydrodynamic inhomogeneity of the flow and the presence of intense vortices in the outlets and the wheel contributes to the fact that the pressure in the vortex regions decreases, provoking the appearance of cavitation.

In such conditions, cavitation and hydrodynamic inhomogeneity of the flow can lead to increased vibration of the pump body parts. As a normalized vibration parameter, the rootmean-square value (RMS) of vibration displacement in the operating frequency band of $10 \ldots 1000 \mathrm{~Hz}$ is taken during stationary operation of the pump. Additionally, the assessment of the frequency components of the vibration spectrum in the characteristic frequency ranges, causally related to the general dynamic state of the unit [17-24].

The distribution of pumps according to the general vibration level, indicating the direction at which the maximum value of vibration displacement is recorded, is presented in Table 1. 
Table 1 -General vibration level of DVL-1400x900 pumps in the $10 \ldots 1000 \mathrm{~Hz}$ band, RMS, $\mu \mathrm{m}$

\begin{tabular}{|c|c|c|c|c|c|c|c|c|}
\hline \multirow{3}{*}{ 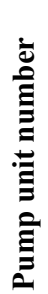 } & \multicolumn{3}{|c|}{ Electric motor } & \multicolumn{5}{|c|}{ Pump } \\
\hline & \multicolumn{3}{|c|}{ Pump installation side } & \multicolumn{2}{|c|}{ Half-coupling side } & \multicolumn{3}{|c|}{$\begin{array}{c}\text { From the stubborn } \\
\text { bearing }\end{array}$} \\
\hline & Horizontal & Vertical & Axial & Horizontal & Vertical & Horizontal & Vertical & Axial \\
\hline 5 & 2.1 & 0.3 & 0.5 & 2.7 & 2.4 & 3.0 & 2.6 & 2.4 \\
\hline 8 & 2.9 & 0.6 & 3.6 & 2.8 & 2.4 & 3.5 & 2.6 & 2.4 \\
\hline
\end{tabular}

As can be seen from Table 1, the overall vibration level of the extreme pump № 8 exceeds the vibration level of the pump № 5. To explain the features of the modes, the hydraulic flow pattern was determined, that is, the boundaries of the transit flow, eddies and stagnant zones.

When the number of operating pumps changed, it was clarified by measurements how the sizes of the vortex and stagnant zones change, the intensity of funnel formation [2,9]. On various units, usually the extreme and one of the middle ones, the distribution of velocities in the water intake and the inlet section of the suction pipes were measured and compared in order to identify the features of the water supply (Fig. 2).
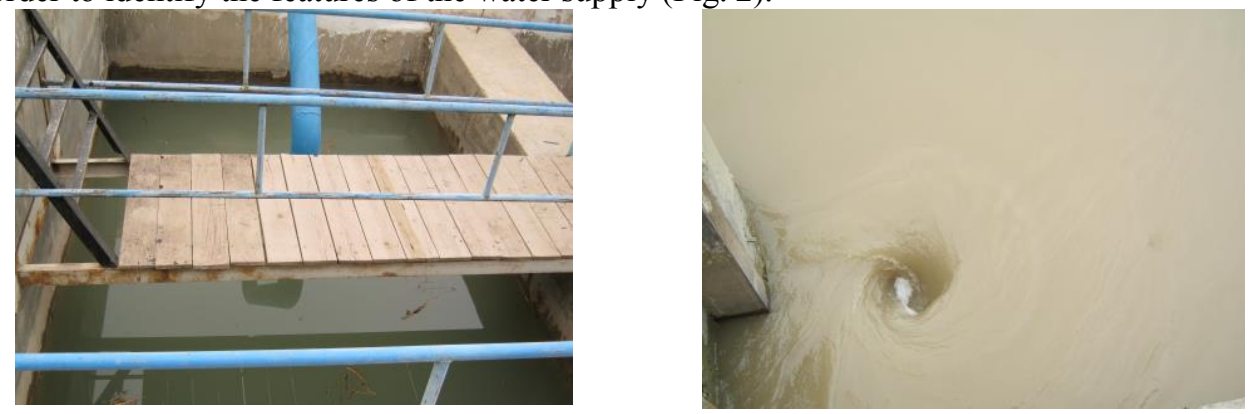

Fig. 2. Studies of the flow supply of the PS "Babotag" in the conditions of funnel formation

Emergency conditions include the operation of pumps with flows that are far beyond the range of nominal flows of pumps of this standard size; during start-up and shutdown periods occurring both in normal operational and emergency situations. In practice, there are cases of their long-term operation with greatly increased, in comparison with the design, hydraulic losses in the suction line, with low water levels in the downstream water level, with mechanical damage to individual elements.

The probability of occurrence of certain deviations from normal operating conditions and the degree of danger of these special modes are different for pumps of different types and are determined by their size, as well as the purpose of the PS, its equipment with control and automation means. For example, an increase in head above the maximum design value poses practically no danger for small centrifugal pumps, but it is highly undesirable for large centrifugal and even more so for axial pumps.

The main difficulties in the operation of pumping and power equipment of large PS are the hydraulic conditions of the flow supply.

The practice of operating the head PS-1 of the largest Karshi cascade, the operation of 46 units at relatively low levels is accompanied by vibration of a cavitation nature. 
Normal operation of the units is achieved by an additional increase in the level of the water horizon in the front chamber (penetration of the impeller).

Inspection of the technical condition of the PS showed that the level in the PS-1 avancamera, which ensures the pump operation without cavitation, should be at least 5.5-6.0 m.

The permissible indicators for the OPV11-260 pumps and the new 300VO-37 / 26C pumps were supposed to be formalized after the end of their trial operation period. Such norms are still absent, and the evaluation criteria set forth in the general technical interstate standards 10816-1-97 and 10816-3-2002 "Vibration. Monitoring the state of the pumping unit based on the results of vibration measurements on non-rotating parts ", where the rootmean-square value of vibration displacement in the operating frequency band of $2 \ldots 1000$ $\mathrm{Hz}$ is set as a normalized parameter of the general vibration level during stationary operation of the pump.

From the point of view of vibration strength in units, the most dangerous are oscillations of a periodic nature, which are the result of mechanical, electromagnetic and hydraulic processes with pronounced discrete components. Such dangerous fluctuations are mainly strong diagnostic signals (that is, they stand out well against the background of vibration noise) [25-27].

In a pump, vibrations of a hydraulic nature are manifested at a blade frequency proportional to the number of blades of the impeller. For the new pump 300VO-37 / 26C there are six of them, and the blade frequency is $f_{b}=6 x$ fo $=25.0 \mathrm{~Hz}$. The OPV11 -260 pump has 4 blades, the vane frequency is $\mathrm{fl}=4 \mathrm{xf}_{\mathrm{o}}=16.67 \mathrm{~Hz}$. The straightening device at the outlet of the impeller excites oscillations with a frequency proportional to the number of its own blades $\mathrm{f}_{\mathrm{a}}=\mathrm{f}_{\mathrm{o}} * 12=50 \mathrm{~Hz}$.

The reason for the non-uniformity of the flow is the asymmetric flow around the rotating blades during the formation of vortex zones. The inhomogeneity of the flow leads to the appearance of vibration on the impeller chamber at the blade frequency $f_{b}$ and its higher harmonics.

For worn out spherical chambers and impellers, in the absence of technological capabilities for turning, a combination of the above reasons prevails.

At the same time, the non-identical operation of the extreme and middle pumps was also established. The uneven approach of water to the extreme units created reverse flows of water in the suction pipes, work with vibrations of modes close to cavitation (Table 2).

Table 2 - Results of comparative vibration measurement $(\mu \mathrm{m})$ of PS-1 KMC units

\begin{tabular}{|c|c|c|c|c|c|}
\hline $\begin{array}{c}\text { Pumping } \\
\text { unit }\end{array}$ & $\begin{array}{c}\text { No } \\
\text { filters }\end{array}$ & $\begin{array}{c}\text { At revolving } \\
\text { frequency }\end{array}$ & $\begin{array}{c}\text { At pole } \\
\text { frequency }\end{array}$ & V mm/s & Notes \\
\hline \multicolumn{7}{|c|}{ Impeller chamber: vertical vibration } \\
\hline Last 6 & $35 \ldots 40$ & $25 \ldots 35$ & 8 & $2,2 \ldots 3,5$ & $\mathrm{P}=8,2 \mathrm{~mW}$ \\
\hline Middle 4 & $17 \ldots 20$ & $13 \ldots 20$ & 4 & $1,5 \ldots 2,2$ & 8,5 \\
\hline \multicolumn{7}{|c|}{ Horizontal } \\
\hline 6 & $60 \ldots 70$ & $26 \ldots 35$ & 24 & $2,7 \ldots 3,2$ & \\
\hline 4 & $62 \ldots 68$ & $17 \ldots 24$ & 21 & $2,8 \ldots 3,2$ & Siphon not charged \\
\hline \multicolumn{7}{|c|}{ Upper motor cross, vertical vibration } \\
\hline 6 & $26 . .35$ & $15 \ldots 16$ & 3 & $1 \ldots 1,3$ & \\
\hline 4 & $10 \ldots 13$ & $2 \ldots 3$ & 1 & 0,5 & \\
\hline \multicolumn{7}{|c|}{ Horizontal } \\
\hline 6 & $76 \ldots 83$ & $61 \ldots 71$ & 4 & $1 \ldots 1,4$ & \\
\hline 4 & $22 \ldots 23$ & $17 \ldots 18$ & 3 & 0,1 & \\
\hline
\end{tabular}


According to the universal characteristic of the modernized pump OP 11-260, we determine the point of intersection of the straight line $\mathrm{H}=$ const with the isoline of the cavitation reserve $\Delta h_{\text {add }}=13,5 \mathrm{~m}$, the abscissa of which gives the maximum flow rate of one unit $[19,20]$.

A new centrifugal pump with changed radii of flow entry into the impeller, blades at the entrance and exit from the impeller was introduced at the Alat pumping station as part of the Amu-Bukhara canal $[4,21]$. The introduction of new pumps reduces losses in the pump flow path and leads to energy savings.

\section{Conclusions}

1. The wear of individual elements leads to a deterioration in the operation of the pumps, a decrease in their efficiency and significant losses. The reasons for the increase in vibration can be the wrong location of the unit in relation to the downstream water level, associated with the peculiarities of their operation, partial mechanical and hydraulic imbalance of the impeller, inhomogeneity of the flow in the impeller. The conducted studies of pumps of large irrigation PS showed a decrease in vibration and an improvement in the main operational parameters during the modernization of the impeller.

2. The modernization of the pump improved its cavitation characteristics by eliminating the center of cavitation at the inlet in the impeller in the area of the driven disk, which increased the reliability and durability of the pump. At the same time, the non-identical operation of the extreme and middle pumps was also established. The uneven approach of water to the extreme units created reverse flows of water in the suction pipes, work with vibrations of modes close to cavitation.

3. Diagnostics of irrigation pumps made it possible to substantiate proposals for the modernization of their main units and operating modes. The distribution of pumps according to the general vibration level is shown, indicating the direction at which the maximum value of vibration displacement was recorded and the results of comparative vibration measurements of PS-1 KMC units.

4. The most rational type of dynamic testing is controlled operation, systematic monitoring of changes in parameters and wear of elements of an operating pump. Under controlled operation, there is no need to accurately measure the absolute values of the parameters; it is necessary to record with high accuracy their time variation and vibration activity. With the participation of the authors, new complex methods of operation are being developed, which allow increasing its reliability by predicting the technical condition.

\section{References}

1. Glovatsky O. Ya., Ergashev R.R. Peculiarities of calculations for reconstruction and modernization of objects of machine water lifting systems. Economic Bulletin Uzb. No. 6, 2009 p. 110-111.(2009)

2. O.Ya. Glovatsky, R.R. Ergashev, Sh.R. Rustamov. New methods of increasing the reliability of the quantitative assessment of the reliability of the operation of objects of machine water lifting systems // International scientific-practical conference "Melioration and problems of restoration of agriculture in Russia" (Kostyakovskie readings). VNIIGiM, Russian Agricultural Academy. -p. 372-376. Moscow, (2013)

3. O.Ya.Glovatskiy, A.B.Saparov, O.R.Azizov Cavitation phenomena of the flow path and impellers of vane pumps // materials of the international scientific-online 
conference on "innovative achievements in science - 2020" Chelyabinsk-Ferghana , , Pp. 521-527. (2020)

4. Oleg Glovatsky, Rustam Ergashev, Azamat Saparov, Mustafo Berdiev and Bobur Shodiev Cavitation-abrasive wear working collectors of pumps // IOP Conference Series: Materials Science and Engineering (2020) 869 04(2006)

5. Beglyarov D.S., Kontsevich I.A., Kozlova M.S., Lentyeva E.A. Methodology for calculating transient processes in pressure water supply systems with pumping stations // Collection of scientific papers. Environmental management and rational use of natural resources are necessary conditions for social and economic development. - M. - S. 47-53. (2005)

6. O.Glovatsky, O.Azizov, F.Bekchanov, A.Gazaryan, M.Shomayramov, N.Ismailov Diagnostic tests of vertical pumps modernized pump stations // International Scientific Conference «Construction Mechanics, Hydraulics and Water Resources Engineering», (CONMEDCHYDRO-2020) IOP Conf. Series: Materials Science and Engineerig, 883(1) 012032 (2020)

7. Platus, D.L. Negative-Stiffness Vibration Isolators. How they Work. - Access mode: https://www.minusk.com/content/technology/how-

itworks_passive_vibration_isolator.html.

8. A.Tokarev, A. Zotov, A. Valeev The application of passive vibroprotective systems having power characteristics with hysteresis loops of rectangular shape for the main pumping units. Dynamics and Vibroacoustics of Machines (DVM2016) // Procedia Engineering. - № 176. - pp. 118 - 127. (2017)

9. Ergashev R.R., Rustamov SH.R., Bekchanov F.A. Increasing to reliability to usages multifunction hydraulic device on system machine ascent of water. 2nd International scientific conference «European Applied Sciences: modern approaches in scientific researches» Volume 3, Stuttgart 18-19th February (2013).

10. Kattakulov, F., Muslimov, T., Khusainov, A., Sharopov, S., Vokhidov, O., Sultanov, S. Water resource saving in irrigation networks through improving the efficiency of reinforced concrete coatings. IOP Conference Series: Materials Science and Engineering.. 883. Pp. 012053. DOI:10.1088/1757-899x/883/1/012053. (2020)

11. Krutov, A., Norkulov, B., Artikbekova, F., Nurmatov, P. Optimal location of an intake at a reservoir prone to salt diffusion. IOP Conference Series: Materials Science and Engineering. 2020. 869. Pp. 072020. DOI:10.1088/1757-899x/869/7/07 (2020).

12. Bazarov, D.R., Mavlyanova, D.A. Numerical studies of long-wave processes in the reaches of hydrosystems and reservoirs. Magazine of Civil Engineering. 87(3). Pp. 123-135. DOI:10.18720/MCE.87.10. (2019)

13. Kan, E., Mukhammadiev, M., Ikramov, N. Methods of regulating the work of units at irrigation pumping stations// FORM-899X/869/4/04 (2009)

14. Bazarov D, Vatin N, Bakhtiyor O, Oybek V. Hydrodynamic effects of the flow on the slab of the stand in the presence of cavitation. IOP Conf Ser Mater Sci Eng;1030:012110. (2021)

15. N. Ismoilov, N. Nasirova, B. Kholbutaev, Kh. Khusanbayeva, O. Nazarov Technology of water supply to water inlets of pumping stations // (IPICSE 2020) IOP Conf. Series: Materials Science and Engineering1030, 012156IOP Publishingdoi:10.1088/1757899X/1030/1/0121561, (2021)

16. Nasrulin A.B., Zhuraev S.R., Saidov F.S. Study of the influence of hydrological and hydraulic parameters on the operating mode of pumping stations / Republican collection of scientific papers, pp. 302-306. Tashkent (2018) 
17. Dilshod Bazarov, Irina Markova BN and OV. Hydraulic aspects of the layout of head structures during water intake from lowland rivers. Ser I O P Conf Sci Mater. 1015:012041. (2021)

18. Panovko, Ya.G. Foundations of the applied theory of vibrations and impact / -L.: Polytechnic, 1990.P. 272.

19. Eshev S, Latipov S, Qurbonov A, Sagdiyev J, Berdiev M, Mamatov N. Non-eroding speed of water flow of channels running in cohesive soils. IOP Conf Ser Mater Sci Eng.;1030:012131. (2021)

20. Kostyukov, V.N., Naumenko A.P. Fundamentals of vibroacoustic diagnostics of machinery // Textbook. - Omsk: SPC "DYNAMIKA", - 283 p. (2006).

21. Khidirov S, Jumaboeva G, Ishankulov Z. Hydraulic mode of operation of the Takhiatash hydroelectric complex. (2021)

22. Barton A.F., Mala-Jetmarova H. An Algorithm for Minimization of Pumping Costs in Water Distribution Systems using a Novel Approach to Pump Scheduling. Mathematical and Computer Modelling, (2017)

23. Obidov B, Vokhidov O, Tadjieva D, Saidkhodjaeva D, Kurbanova U, Isakov A. Hydrodynamic effects on the flow elements of the downstream devices in the presence of cavitation. IOP Conf Ser Mater Sci Eng. 1030:012114. (2021)

24. Jong-Woong Choi, Young-Do Choi, Chang-Goo Kim, Young-Ho Lee Flow uniformity in a multi-intake pump sump model / Journal of Mechanical Science and Technology 2010, Volume 24, Issue 7. Peking.

25. Wittkoff Wallace. Efficiency through indirect measures/ World Pumps. New York, (2012)

26. Krutov A, Norkulov B, Uljaev F, Jamalov F. Results of a numerical study of currents in the vicinity of a damless water intake. IOP Conf Ser Mater Sci Eng. 1030:012121. (2021)

27. Matyakubov B, Begmatov I, Raimova I, Teplova G. Factors for the efficient use of water distribution facilities. IOP Conf Ser Mater Sci Eng. 883:012025. (2020) 